\title{
Les pratiques phytosanitaires des producteurs de légumes de l'île Maurice: impacts et perspectives de changement
}

\author{
Fabrice Le Bellec ${ }^{1, *}$, Maud Scorbiac ${ }^{2}$ et Jacqueline Sauzier ${ }^{2}$ \\ ${ }^{1}$ CIRAD, UPR HORTSYS, 97455 Saint-Pierre, La Réunion, France \\ ${ }^{2}$ Mauritius Chamber of Agriculture, Vivéa Business Park, Block 18 Bâtiment 1827, Saint Pierre, Mauritius
}

\begin{abstract}
Résumé - L'utilisation de pesticides n'est pas sans impact sur l'environnement ni sur la santé humaine. La société civile mauricienne s'est récemment emparée de la question suite à des détections de pesticides dans des légumes prélevés dans les marchés de l'île. Mais quelles sont les marges de manœuvre des producteurs pour faire face à cette demande sociétale ? Comme les méthodes de production étaient jusqu'ici peu documentées, nous avons mené une enquête auprès de 298 producteurs de légumes afin d'analyser leurs pratiques phytosanitaires et d'en évaluer les impacts sur l'environnement. Cette enquête vise à répondre aux questions suivantes : Quels sont réellement les usages de pesticides des producteurs? Quelles contraintes supportent-ils? Les producteurs sont plutôt âgés (60\% ont plus de 50 ans) et peu d'entre eux ont une formation initiale de base. Près des deux tiers d'entre eux ne sont pas propriétaire du foncier. Dans ces conditions, les producteurs pratiquent une agriculture d'opportunisme visant à optimiser leur parcelle dans le temps et dans l'espace. Aucun risque, aucune perte. La grande majorité des producteurs protègent leurs cultures par des traitements phytosanitaires préventifs contre les nombreux bio-agresseurs recensés. Entre 11 et 53 traitements par an sont ainsi effectués selon les successions culturales entreprises. Près de 60 insecticides et fongicides sont couramment utilisés par les producteurs. L'outil d'aide à la décision «Phyto'Aide» nous a permis d'évaluer, ex ante, le risque du transfert de ces substances vers l'environnement. Ces risques s'avèrent importants pour tous les pesticides car les bonnes pratiques phytosanitaires sont peu ou pas respectées par les producteurs. Dans ces conditions, de nouvelles stratégies de protection contre les bioagresseurs s'avèrent nécessaires. Nous proposons dans cet article différentes pistes d'amélioration, mais aussi une stratégie d'accompagnement de ces producteurs pour faire face à une demande sociétale d'une production saine et sûre.
\end{abstract}

Mots clés : pesticide / pratique agricole / enquête sur exploitation agricole / évaluation environnementale / culture maraîchère

\begin{abstract}
Phytosanitary practices of vegetable growers in Mauritius: impact and prospects for change. The use of pesticides for pest control has an impact on both environment and human health. In 2015, the Mauritian government expressed the wish to develop organic farming which further spiked the interest of the local community following the detection of pesticides residues in samples sourced from different vegetable markets across the Island. But to what extent can producers respond to this request from the local society? Given that production methods were poorly documented, we surveyed 298 vegetable growers (beans, cabbages, tomatoes, potatoes, cucurbits, etc.) in order to analyze their phytosanitary practices and evaluate their impact on environment. The purpose of this survey is to provide answers to the following questions: How do producers actually use pesticides? What constraints do they face? Producers are rather old ( $60 \%$ are over 50 years old) and few have basic training which is rarely specific to agriculture. Moreover, almost two-thirds of the respondents do not own the land they cultivate which in turn leads them to adopt a risk averse position and focus on maximising their gains - monetary or otherwise - from their plot over a span of time. The majority of producers have recourse to preventive treatments to protect their crops against the numerous pests identified. Between 11 and 53 treatments per year are thus carried out depending on the crop rotation patterns. Nearly 60 insecticides and fungicides are commonly used by producers. "Phyto'Aide", a tool which aims at facilitating the decision-making process, allowed us to evaluate,
\end{abstract}

$\overline{{ }^{*} \text { Auteur de correspondance }}$ : fabrice.le_bellec@cirad.fr 
ex ante, the risk of transferring these substances to the environment. It turned out that risks are important for all pesticides because good phytosanitary practices are inadequately, if at all, followed by most producers. Under these conditions, new strategies for crop protection against pests are needed. We propose in this article different clues for improvement but also a strategy to assist these producers in responding to society's demand for a safe and healthy production.

Keywords: pesticides / agricultural practices / farm surveys / environmental assessment / vegetable crops

\section{Introduction}

À l'île Maurice, des taux hors normes de résidus de pesticides sont régulièrement détectés dans les fruits et légumes prélevés sur les marchés. En 2013, près de $38 \%$ des 418 échantillons présentaient des traces de pesticides, dont un peu plus de $7 \%$ s'avéraient être au-dessus des seuils légaux (Boodhoo, 2014). Ces taux étaient environ trois fois inférieurs en 2010; cette évolution préjuge donc d'une situation préoccupante. La société civile mauricienne s'est d'ailleurs emparée de cette question et, par voie de presse, a interpellé les producteurs, car «il y va de la santé et de la salubrité publiques» (Forget et al., 2016). Cette défiance des consommateurs vis-à-vis des producteurs a très rapidement engendré une réponse politique communiquée lors du dernier discours du ministre des Finances: "To encourage the production of bio food, we are introducing a Bio Farming Development Certificate (..). We have the ambition of producing no less than 50 per cent of our total local food production according to bio norms $»$. (Lutchmeenaraidoo, 2016). Les producteurs mauriciens peuvent-ils faire face à ces nouveaux enjeux sociétaux? Même si la culture de la canne à sucre reste le pilier de l'agriculture mauricienne (près de $51000 \mathrm{ha}$ ), les productions agricoles tendent à se diversifier, notamment par le maraîchage. Ainsi, en 2014, les cultures maraîchères s'étendaient sur près de 8500 ha, employant près de 12000 producteurs. Ces productions jouent un rôle important dans le développement endogène de l'île Maurice qui vise l'autosuffisance alimentaire en légumes frais. La filière reste cependant informelle et peu organisée, bien que les producteurs bénéficient d'un appui technique du Food and Agricultural Research and Extension Institute (FAREI) pour les aider à produire. Malgré cela, près de $2000 \mathrm{t}$ de pesticides sont importées à l'île Maurice chaque année, dont plus de $50 \%$ sont des insecticides et des fongicides à usage agricole (Boodhoo, 2014), qui sont essentiellement utilisés en cultures maraîchères. Les herbicides représentant l'autre moitié de ces importations sont principalement utilisés en culture de canne à sucre. Les conditions pédoclimatiques tropicales de l'île Maurice, particulièrement favorables aux bioagresseurs, sont à l'origine de l'usage de ces pesticides. En effet, des développements fulgurants de maladies et des changements de statut des ravageurs sont souvent observés en milieu tropical (Cilas et al., 2015). Face à ces phénomènes, seuls sont employés des moyens de lutte chimique, souvent en urgence et sans garantie d'efficacité, notamment à cause de résistances de certains bioagresseurs aux pesticides. Face à toutes ces pressions, l'objectif de notre étude est donc d'analyser les pratiques phytosanitaires actuelles des producteurs de légumes mauriciens, afin d'identifier à quelles contraintes ils sont soumis et quelles réponses ils apportent. Nous évaluerons ces pratiques du point de vue environnemental. Enfin, nous discuterons des moyens pour limiter les risques liés aux usages de ces pesticides et des méthodes d'accompagnement pour y parvenir.

\section{Matériel et méthode}

\subsection{Enquête réalisée auprès des producteurs}

L'enquête a été réalisée d'août à novembre 2015 auprès de 298 producteurs des 11 principaux légumes produits à l'île Maurice. Quatre sous-échantillons ont été déterminés proportionnellement aux surfaces de ces 11 cultures légumières des quatre grands bassins de production de l'île (Fig. 1). Ainsi, 93 producteurs ont été interrogés dans le Nord, 82 dans l'Est, 78 dans le Centre-Ouest et 46 dans le Sud. Quatorze techniciens d'encadrement du FAREI (3 ou 4 par bassin de production) nous ont mis en contact avec ces producteurs et ont également apporté leur appui durant les enquêtes (traduction lorsque cela était nécessaire, précision de termes techniques, identification des bio-agresseurs...). Les entretiens semidirectifs ont comporté 57 variables quantitatives et qualitatives articulées autour de quatre grands thèmes : l'exploitant et son exploitation, les pratiques culturales de la culture principale de l'exploitant, la mise en œuvre de pratiques alternatives aux pesticides et les perspectives de l'exploitant (Tab. 1). La culture principale étudiée lors des entretiens a été proposée par le producteur lui-même. Ainsi, sur nos 298 agriculteurs, 99 se considèrent plutôt spécialistes des cucurbitacées (Cucurbita spp.), $48 \mathrm{du}$ haricot vert (Phaseolus vulgaris), 34 des choux (Brassica spp.), 31 de la tomate (Solanum lycopersicum), $31 \mathrm{de}$ la pomme de terre (Solanum tuberosum), 14 des piments (Capsicum spp.), 12 des oignons (Allium cepa), 9 des aubergines (Solanum melongena), 8 des légumes «feuilles et/ou aromates» comprenant les salades (Lactuca spp.), la coriandre (Coriandrum sativum), le thym (Thymus vulgaris), $7 \mathrm{du}$ lalo ou gombo (Abelmoschus esculentus) et 5 de la carotte (Daucus carota subs. sativus).

\section{2 Évaluation ex ante des performances environnementales du système de culture}

Nous avons évalué les performances environnementales du système de culture à l'aide de l'indice de fréquence de traitement (IFT) et de l'outil d'aide à la décision Phyto'Aide (http://www.margouilla.net/phytoaide).

L'indice de fréquence de traitement (IFT) est calculé selon la formule suivante:

$$
\text { IFT }=\frac{\begin{array}{c}
\text { dose de produit commercial appliqué } \\
\text { sur la parcelle } \times \text { surface traitée })
\end{array}}{\begin{array}{c}
\text { dose homologuée de produit } \\
\text { commercial } \times \text { surface de la parcelle })
\end{array}} .
$$




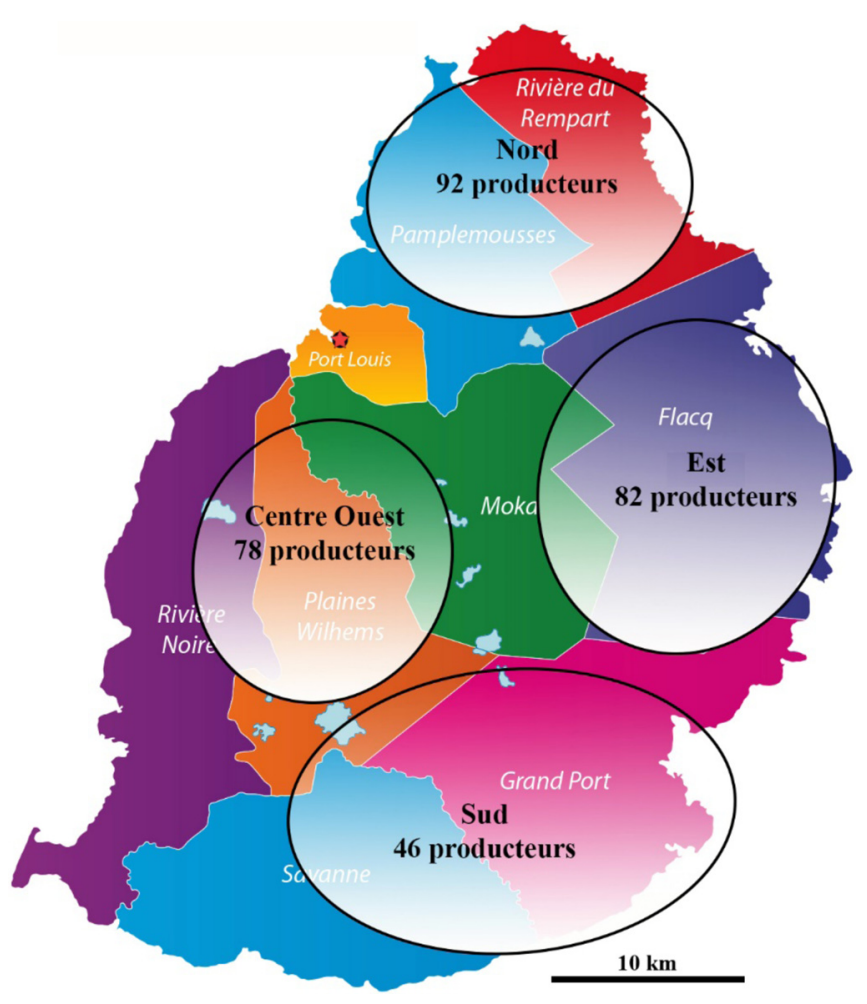

Fig. 1. Carte de l'île Maurice et répartition des producteurs interrogés par bassin de production (Nord, Est, Centre-Ouest et Sud).

Fig. 1. Map of Mauritius and distribution of producers surveyed by production basin (North, East, Central-West and South).
L'IFT est un indicateur de suivi de l'utilisation des pesticides. Il comptabilise le nombre de doses de référence utilisées sur le système de culture, ce qui permet de situer les pratiques d'un producteur par rapport à un autre mais aussi de comparer les systèmes de culture entre eux.

Nous avons utilisé l'outil d'aide à la décision « Phyto'Aide » pour réaliser une évaluation ex ante des risques de transfert vers l'environnement des produits phytosanitaires utilisés par les producteurs mauriciens. "Phyto'Aide» repose sur les résultats de l'indicateur I-Phy (Bockstaller et al., 2008; Van der Werf et Zimmer, 1998). I-Phy considère ces risques en fonction des caractéristiques du pesticide appliqué et des conditions d'utilisation de ce dernier. "Phyto'Aide» simule des scénarios d'utilisation d'un pesticide en faisant varier dix variables d'utilisation selon leurs deux attributs (conditions d'utilisation favorable et défavorable), soit $10^{2}$ scénarios (voir Tab. 2). Chaque scénario simulé aboutit à un score I-Phy. Celui-ci correspond à un score de risque de transfert du pesticide renseigné sur une échelle qualitative de 0 à 10 , où un score supérieur à 7 est considéré comme «acceptable» en termes de risque de transfert. L'application «Phyto'Aide» propose les scores I-Phy minimum et I-Phy maximum de tous les scénarios testés (variables nommées: I-Phy_min et I-Phy_max). I-Phy_min correspond au scénario d'utilisation du pesticide le plus défavorable en termes de risque de transfert. Comme par exemple, utiliser un appareil de traitement de type «atomiseur» à proximité (moins de 5 mètres) d'un point d'eau; dans ces conditions, un produit phytosanitaire à base de mancozèbe obtiendra un score proche de 4 sur cette échelle de 0 à 10. A contrario, I-Phy max correspond au scénario d'utilisation du pesticide le plus favorable en termes de risque de

Table 1. Liste des 57 variables d'enquête.

Table 1. List of the 57 variables using for the survey.

\begin{tabular}{|c|c|c|c|}
\hline $\begin{array}{l}\text { L'exploitant et son exploitation } \\
\text { (15 variables) }\end{array}$ & $\begin{array}{l}\text { Pratiques culturales et connaissance } \\
\text { des impacts } \\
\text { (21 variables) }\end{array}$ & $\begin{array}{l}\text { Pratiques alternatives } \\
\text { (10 variables) }\end{array}$ & $\begin{array}{l}\text { Perspectives de l'exploitant } \\
\text { (11 variables) }\end{array}$ \\
\hline
\end{tabular}


Tableau 2. Liste des dix variables d'utilisation d'un pesticide et leurs deux attributs (conditions d'utilisation favorable et défavorable) utilisées par «Phyto'Aide» pour construire ses scénarios.

Table 2. List of 10 variables for using a pesticide and their two attributes (favorable and unfavorable conditions) used by 'Phyto'Aide' to build its scenarios.

\begin{tabular}{|c|c|c|}
\hline Variables & $\begin{array}{l}\text { Classe } \\
\text { favorable }\end{array}$ & $\begin{array}{l}\text { Classe } \\
\text { défavorable }\end{array}$ \\
\hline $\begin{array}{l}\text { 1. Distance de la parcelle à un point } \\
\text { d'eau }\end{array}$ & $>15 \mathrm{~m}$ & $<5 \mathrm{~m}$ \\
\hline 2. État du point d'eau & $\mathrm{Sec}$ & En eau \\
\hline 3. Saison du traitement & Sèche & Humide \\
\hline $\begin{array}{l}\text { 4. Nombre de jours sans pluie } \\
(>5 \mathrm{~mm})\end{array}$ & $>30$ & $<3$ \\
\hline 5. État de couverture du sol & Total & Sol nu ou partiel \\
\hline $\begin{array}{l}\text { 6. Bande enherbée non cultivée en } \\
\text { bas de parcelle }(\mathrm{m})\end{array}$ & Oui & Non \\
\hline 7. Type de pulvérisateur & Autorégulé & Atomiseur \\
\hline 8. Type de buse du pulvérisateur & Antidérive & Fente classique \\
\hline $\begin{array}{l}\text { 9. Cache anti-dérive } \\
\text { 10. Position d'application du } \\
\text { pesticide }\end{array}$ & Oui & Non \\
\hline 10. a) cas des herbicides & $\begin{array}{l}\text { Désherbage } \\
\text { localisé }\end{array}$ & Sur sol nu \\
\hline $\begin{array}{l}\text { 10. b) autres pesticides (nombre de } \\
\text { jours entre le traitement et la } \\
\text { dernière opération } \\
\text { d'entretien de l'enherbement } \\
\text { ou du couvert) }\end{array}$ & $>20$ & $<20$ \\
\hline
\end{tabular}

transfert vers l'environnement. Comme par exemple, utiliser un appareil de traitement de type «autorégulé» équipé d'un cache antidérive et à plus de 15 mètres d'un point d'eau; dans ces conditions, ce même produit phytosanitaire à base de mancozèbe obtiendra un score proche de 7,5 sur cette échelle de 0 à 10. La différence entre ces scores I-Phy_max et I-Phy_min constitue la marge de progrès possible (passage de la condition défavorable à favorable des variables). "Phyto'Aide» détermine les variables permettant de limiter ces risques de transfert. Une fois identifiées, nous avons confronté ces variables, dites de contribution du score I-Phy, aux données issues de l'enquête (pratiques phytosanitaires et caractéristiques de la parcelle) pour déterminer si les conditions d'utilisation des pesticides par les producteurs risquaient ou pas d'accentuer ces transferts. Pour réaliser cette analyse, nous avons évalué une à une toutes les substances actives des pesticides utilisées par les producteurs. Cependant, pour simplifier cette analyse, nous avons évalué ces risques sur un seul type de sol afin de les comparer entre elles. Les caractéristiques de sol pour réaliser cette étude sont les suivantes: sol d'une profondeur supérieure à $20 \mathrm{~cm}$, d'un $\mathrm{pH}$ compris entre 5 et 5,5, argileux et avec présence d'argile gonflante, avec un taux de matière organique compris entre 2 et $5 \%$, non filtrant et avec une pente comprise entre 5 et $10 \%$.

\subsection{Analyses statistiques}

Les analyses statistiques ont été réalisées avec le logiciel $\mathrm{R}$ (Version 3.2.2, R Development Core Team 2013, Vienna, Austria). Nous avons cherché à expliquer les variables «IFT»,
« respect ou non des bonnes pratiques agricoles » (testées une à une, Tab. 1, colonne 2) et «mise en œuvre des méthodes alternatives » (Tab. 1, colonne 3) en fonction de l'âge et du niveau de formation du producteur, mais aussi du bassin de production, de la superficie de l'exploitation, du niveau d'équipement agricole et du statut foncier. La dépendance des différentes variables entre elles a été testée à l'aide d'un test de $\mathrm{khi}^{2}$. Nous avons réalisé une analyse en composantes principales (ACP), suivie d'une classification ascendante hiérarchique $(\mathrm{CAH})$, afin de regrouper les pesticides ayant les mêmes variables de contribution du score I-Phy. L'ACP et la CAH ont été réalisées avec le package «factorMinR». Enfin, les différences des moyennes d'IFT par légume ont été testées à l'aide des tests de Kruskall-Wallis et de Wilcoxon au seuil de $5 \%$.

\section{Résultats}

\subsection{Typologie des producteurs}

Pour $86 \%$ des producteurs interrogés, l'agriculture est l'activité principale ou unique. Ces producteurs sont exclusivement des maraîchers (90\%), sans qu'ils soient pour autant spécialisés sur une seule culture légumière. Lorsqu'une activité agricole complémentaire existe, ils sont producteurs de canne à sucre, de fruits, de thé ou encore éleveurs. La taille moyenne des parcelles exploitées est de 1,5 ha. La majorité $(56 \%)$ des producteurs cultivent entre 0,5 et 2 ha, tandis que $14 \%$ cultivent moins de 0,5 ha et $30 \%$ plus de 2 ha. Seulement $37 \%$ des agriculteurs sont propriétaires des terres qu'ils exploitent, les autres sont liés à un tiers propriétaire (agriculteur ou pas) par un bail écrit pour une durée d'au moins un an $(38 \%)$, ou une durée inférieure à un an $(5 \%)$ ou encore sans aucune garantie (seulement un accord oral de cultiver), ces derniers représentant $20 \%$ de l'échantillon. Près de $60 \%$ des producteurs interrogés ont plus de 50 ans. Les compétences agricoles ont été acquises la plupart du temps grâce à un aïeul ; ils sont d'ailleurs peu nombreux à avoir suivi des études spécialisées agricoles (5\%). Par contre, les producteurs se tiennent informés et complètent leurs connaissances grâce aux formations organisées par le FAREI. Même si les surfaces cultivées sont faibles, la production de légumes nécessite une main-d'œuvre importante. En effet, peu d'opérations culturales sont actuellement mécanisées, le plus souvent faute de moyens financiers. Quelques producteurs mobilisent de la main-d'œuvre familiale (18\%), tandis que la plupart des autres emploient des salariés soit à l'année (pour $40 \%$ d'entre eux), soit occasionnellement lorsque la charge de travail est plus importante (pour 52\% d'entre eux). L'objectif premier de ces producteurs est de garantir la productivité de leurs cultures. Cependant, près de $90 \%$ des producteurs interrogés estiment qu'il est nécessaire de changer leur façon de produire en réponse à la demande sociétale. Ils expriment pour cela des besoins d'encadrement et de formation pour les y aider.

\subsection{Bioagresseurs des cultures et leur gestion}

Les producteurs ont rapporté 24 principaux bioagresseurs sur leurs cultures. Les cultures de cucurbitacées, de tomates et de pommes de terre sont les plus affectées en nombre d'espèces, avec plus de dix bioagresseurs chacune. Aux dires des producteurs, deux bioagresseurs sont aujourd'hui pré- 
gnants et de contrôle difficile: une mouche dont la larve occasionne des galeries sur les feuilles et les mouches affectant les fruits des cucurbitacées. Ces bioagresseurs ont été identifiés par les techniciens du laboratoire d'entomologie du FAREI comme étant Liriomyza trifolii pour la mouche-mineuse et Bactrocera cucurbitae, Dacus ciliatus et Dacus demmerezi pour les mouches des cucurbitacées. Liriomyza trifolii s'attaque à toutes les cultures sans exception, les mouches à toutes les cucurbitacées comme les citrouilles ou les courgettes. Pour faire face à ces bioagresseurs, $90 \%$ des producteurs ont une stratégie de protection préventive (déclenchement des traitements à un pas de temps régulier). Nous avons relevé le nom de 132 spécialités commerciales de produits phytosanitaires utilisées par les producteurs. Celles-ci correspondent à 67 substances actives différentes, dont notamment 30 insecticides et acaricides comme l'abamectine, la cypermethrine, la cyromazine ou la chlorantraniliprole, et 26 substances actives fongicides comme le métalaxyl, le mancozèbe ou l'hydroxyde de cuivre. Onze substances actives herbicides ont aussi été rapportées, le glyphosate et le paraquat restant les plus utilisés. La plupart des spécialités commerciales sont constituées d'une seule substance active, seules sept d'entre elles sont composées de deux (6 spécialités commerciales associent 2 substances actives fongicides et une seule est composée de 2 substances actives insecticides). Le Tableau 3 (colonnes 1 à 3 ) reprend la liste des substances actives correspondant aux spécialités commerciales citées par au moins 10 producteurs, soit 37 substances actives. Peu de producteurs mettent en œuvre une traçabilité complète de leurs opérations de traitement et de récolte, ce qui ne nous a pas permis de calculer des IFT réels. Cependant, comme beaucoup d'entre eux (270 sur 298) réalisent des traitements réguliers, nous avons calculé, sur cet échantillon de 270 producteurs, des IFT théoriques par culture en considérant leurs fréquences individuelles de traitement (entre 7 et 14 jours) et en s'appuyant sur des durées moyennes de cycle de culture excluant les périodes de récolte. Trois catégories d'IFT se distinguent (Tab. 4) :

- IFT élevés : cultures d'aubergines et de tomates;

- IFT moyens: culture d'oignons, de cucurbitacées et de choux;

- IFT faibles : culture de légumes feuilles, de haricots et de carottes.

L'IFT de la culture du piment ne se distingue pas statiquement des IFT moyens et élevés. De même, l'IFT de la pomme de terre ne se distingue pas statiquement des IFT faibles et moyens. Aucune relation entre ces IFT, les bassins de production, les planteurs (âge, formation), le statut du foncier et le niveau d'équipement n'a pu être mise en évidence. Pour évaluer et comparer une pression annuelle liée à l'usage des pesticides d'une parcelle à l'autre, nous avons cumulé les IFT des successions culturales constatées chez les producteurs. En considérant l'ensemble des bassins de production, 15 successions culturales annuelles différentes ont été rapportées par les producteurs. Les cumuls d'IFT varient quant à eux de 11 à 53 selon ces successions (Tab. 5). Ce maximum est observé lorsqu'un cycle de cucurbitacées est inséré entre deux cycles de tomates ou lorsqu'un cycle de haricot est inséré entre deux cycles de cucurbitacées.

\subsection{Bonnes pratiques agricoles (BPA) et techniques alternatives à l'usage des pesticides}

Une très grande majorité de producteurs $(95 \%)$ observent les ravageurs de leur culture et sont capables de faire un choix pertinent $\mathrm{du}$ pesticide à utiliser vis-à-vis de sa cible. Cependant, le déclenchement du traitement est décidé sans considérer un seuil de nuisibilité de ces ravageurs. Les traitements sont effectués sans prise en considération des conditions climatiques, des insectes utiles (traitement pendant les floraisons) ou du voisinage. Globalement, peu de producteurs mettent en place des techniques permettant d'éviter l'usage des pesticides d'origine chimique. De rares expériences ont cependant été rapportées comme, par exemple, l'utilisation de plantes pièges ou répulsives (des capucines pour attirer les pucerons et des œillets d'Inde pour repousser les fourmis par $20 \%$ des producteurs), les jachères améliorantes (cycle de haricots pour enrichir le sol en azote par $10 \%$ des producteurs), le lâcher d'auxiliaires des cultures (des coccinelles fournis par le FAREI par $15 \%$ des producteurs) ou bien encore la création d'habitats refuge pour ces mêmes auxiliaires ( 1 unique producteur a aménagé des bandes fleuries [espèces végétales spontanées et du maïs] sur le pourtour de sa parcelle). La rotation des cultures est pratiquée par près de $80 \%$ des producteurs, même s'ils ne la considèrent pas réellement comme une pratique conduisant au non-usage des pesticides. En effet, ces rotations ne sont que des alternances annuelles de cultures de légumes (Tab. 5); dans ces conditions, ces rotations sont de l'ordre de l'optimisation de l'occupation des sols et/ou de l'adéquation du type de production avec son marché ou sa saisonnalité. Ni l'âge du producteur (valeur de $p=0,68$ ), ni le nombre d'années d'expérience (valeur de $p=0,49$ ), ni le niveau de formation (valeur de $p=0,12$ ), ni l'origine des compétences acquises en agriculture (valeur de $p=0,38)$ n'influencent le respect des BPA ou l'adoption des techniques alternatives à l'usage des pesticides. Par contre, la sécurité foncière (producteurs propriétaires ou avec un bail d'une durée supérieur à 1 an) influence favorablement les BPA (valeur de $p=0,045$ ) et même l'adoption de pratiques alternatives (valeur de $p=0,00001$ ).

\section{4 Évaluation des risques de transfert des pesticides vers l'environnement}

Le Tableau 3 (colonnes 4 à 6) reprend les scores I-Phy_min et I-Phy_max de l'ensemble des pesticides les plus utilisés par les producteurs. Selon la substance active, ces scores varient de 4,3 pour deux fongicides (le mancozèbe et le chlorothalonile) à 9,8 pour un fongicide (le difénoconazole) et un insectide (l'acetamiprid). Hormis trois substances actives (l'acetamiprid, la deltamethrine et l'émamectine benzoate), toutes les autres substances ont un score I-phy min inférieur à 7 si elles sont utilisées dans de mauvaises conditions. Cependant, toutes les substances actives ont une marge de progrès leur permettant d'atteindre ou de dépasser ce score de 7 si les bonnes conditions d'utilisation sont toutes observées. L'analyse en composantes principales a permis d'identifier les principales variables d'importance aux scores de chaque substance active : la distance du point d'eau et son état, le type de pulvérisateur utilisé et la position d'application du pesticide. Selon la 
Tableau 3. Liste des principales substances actives répertoriées durant l'enquête de terrain auprès de 298 producteurs de l'île Maurice. Table. List of the main active substances established during the field survey of 298 producers in Mauritius.

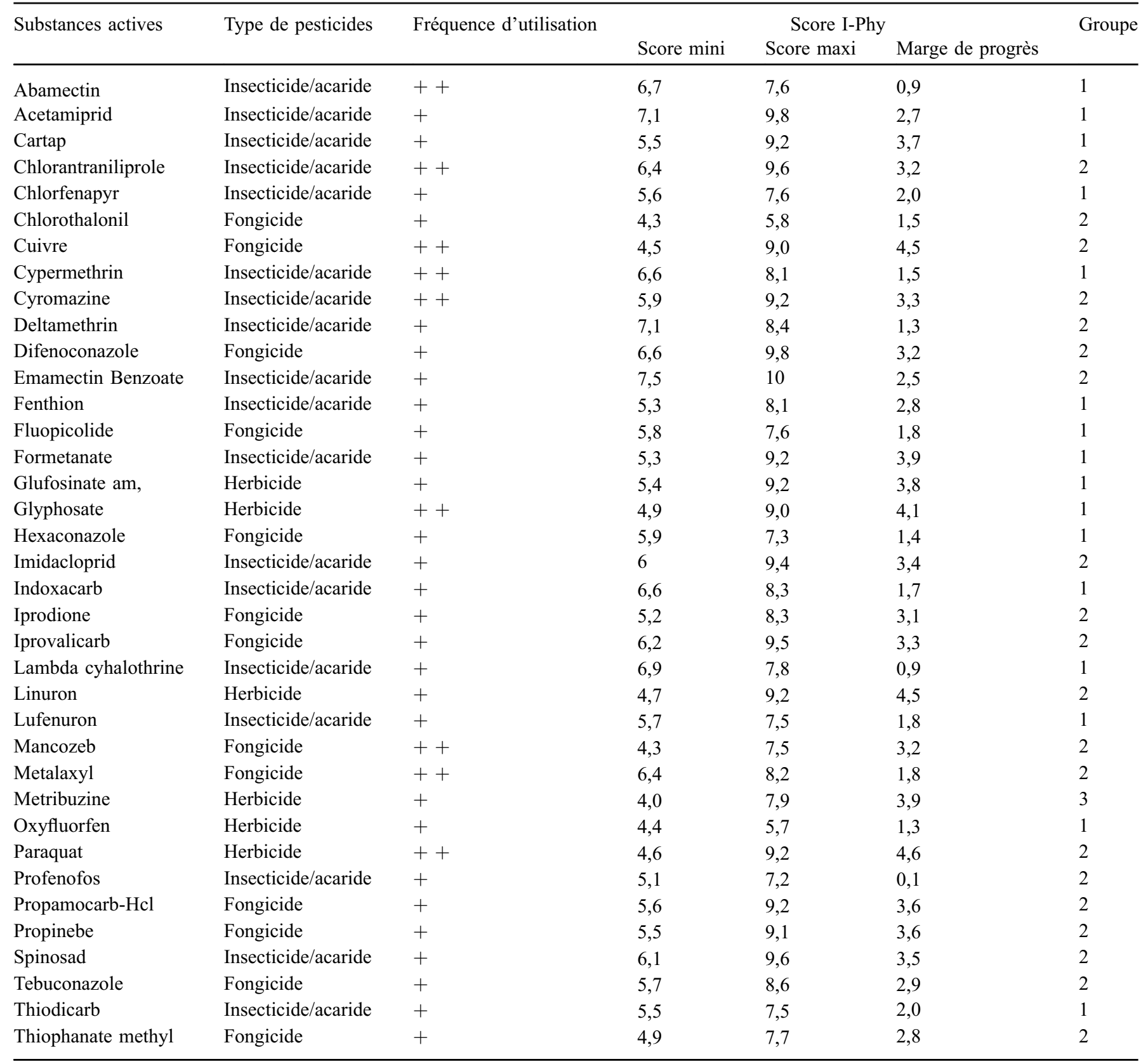

Fréquence d'utilisation : + + (par plus de 100 producteurs) + (entre 10 et 100 producteurs). Le score I-Phy correspond à un risque de transfert du pesticide sur une échelle qualitative de 0 à 10 , où un score de 7 et plus est considéré comme « acceptable ». I-Phy_min correspond au score des conditions d'utilisation défavorable du pesticide. I-Phy_max correspond au score des conditions d'utilisation favorable du pesticide. Marge de progrès $=($ I-Phy max-I-Phy_min). Groupe $1:$ substances actives pour lesquelles la distance au point d'eau et l'état de ce point d'eau ont une importance majeure dans la construction du score I-Phy. Groupe 2 : substances actives pour lesquelles le type de pulvérisateur a une importance majeure dans la construction du score I-Phy. Groupe 3 : substances actives pour lesquelles la position d'application a une importance majeure dans la construction du score (voir aussi Tab. 1).

Frequency of use: ++ (by more than 100 producers), + (between 10 and 100 producers). I-Phy score corresponds to a risk of transfer of the pesticide on a qualitative scale of 0 to 10 where a score of 7 and more is considered as 'acceptable'. I-Phy_min corresponds to the score of the unfavorable conditions for using a pesticide. I-Phy_max corresponds to the score of favorable conditions for using the pesticide. Margin of progress $=($ I-Phy max-I-Phy_min). Group 1: active substances for which the distance to the water point and the state of this water point are of major importance in the construction of the I-Phy score. Group 2: active substances for which the type of sprayer is of major importance in the construction of the I-Phy score. Group 3: active substances for which the application position is of major importance in the construction of the score (see also Tab. 1). 
substance active, ces variables n'ont pas le même ordre d'importance. La classification hiérarchique ascendante a permis de classer les substances actives en trois groupes; leur appartenance à chacun des groupes est indiquée dans le Tableau 3 (colonne 7):

Tableau 4. Légumes associés à leur Indice de fréquence de traitement (IFT) moyen et leur écart-type déduits de l'ensemble des IFT pour chaque légume et classés selon leur intensification de traitement.

Table 4. Vegetables related to their average Frequency of Treatment Index (FTI) and standard deviation deduced from all FTIs for each vegetable and classified according to their intensification of treatment.

\begin{tabular}{lll}
\hline Légumes & IFT Moyen (écart-type) & \\
\hline Aubergine & $23,62( \pm 5,42)$ & $\mathrm{a}$ \\
Tomate & $23,25( \pm 5,29)$ & $\mathrm{a}$ \\
Piment & $19,03( \pm 2,8)$ & $\mathrm{ab}$ \\
Oignon & $14,92( \pm 4,86)$ & $\mathrm{b}$ \\
Cucurbitacées & $14,65( \pm 7,08)$ & $\mathrm{b}$ \\
Choux & $12,93( \pm 5,52)$ & $\mathrm{b}$ \\
Pomme de terre & $8,96( \pm 1,49)$ & $\mathrm{bc}$ \\
Lalo & $8,3( \pm 1,8)$ & $\mathrm{c}$ \\
Légumes feuilles & $7,4( \pm 2,02)$ & $\mathrm{c}$ \\
Haricot & $7,33( \pm 2,94)$ & $\mathrm{c}$ \\
Carotte & $4,5( \pm 1,56)$ & $\mathrm{c}$ \\
\hline
\end{tabular}

$\mathrm{a}=$ IFT élevés, $\mathrm{b}=$ IFT moyens, $\mathrm{c}=$ IFT faibles, $\mathrm{ab}$ et $\mathrm{bc}$ correspondant aux légumes intermédiaires à deux catégories. Significativité déduite par les tests de Kruskall-Wallis et de Wilcoxon au seuil de $5 \%$, $\mathrm{a}=$ heavily treated, $\mathrm{b}=$ moderately treated, $\mathrm{c}=$ little treated, $\mathrm{ab}$ and $\mathrm{bc}$ corresponding to intermediate vegetables in two categories. Significance deduced by the Kruskall-Wallis and Wilcoxon tests at the $5 \%$ threshold.
- groupe 1 : substances actives pour lesquelles la distance et l'état du point d'eau ont une importance majeure dans la construction du score I-Phy; puis suit, dans une moindre mesure, le type de pulvérisateur utilisé. Des points d'eau avec des cultures souvent très proches ont été observés dans près d' $1 / 3$ des parcelles des producteurs interrogés. Les substances actives du groupe 1 présentent des risques importants de transfert vers ces points d'eau, notamment lorsque les conditions d'application (BPA) ne sont pas respectées. Le glyphosate, l'herbicide le plus utilisé par les producteurs, ou encore des insecticides comme le cartap ou le fenthion, dont les scores I-Phy_min sont respectivement 5,5 et 5,3, font craindre alors des risques de transfert importants;

- groupe 2: substances actives pour lesquelles le type de pulvérisateur a une importance majeure dans la construction du score I-Phy; puis suivent, dans une moindre mesure, la distance et l'état du point d'eau. Les substances actives du groupe 2-et notamment le mancozèbe (I-Phy_min de 4,3 et I-Phy_max de 7,5), l'un des fongicides les plus utilisés par les producteurs - sont particulièrement concernées par ces risques. Près de $60 \%$ des producteurs utilisent des appareils de traitement de type atomiseur qui, sans dispositif antidérive et les BPA respectées, présentent des risques importants de transfert des pesticides vers l'air (Butler-Ellis et al., 2002);

- groupe 3: substances actives pour lesquelles la position d'application a une importance majeure dans la construction du score I-Phy; puis suivent, dans une moindre mesure, le type de pulvérisateur, la distance et l'état du point d'eau. Ce groupe concerne principalement des herbicides, et en particulier la metribuzine. Utilisées sur sol nu (usage en prélevée), les substances actives de ce groupe présentent des risques importants de transfert notamment si des pluies (ou une irrigation) interviennent peu après le traitement (Davis et al., 2011).

Tableau 5. Successions culturales sur une année constatées chez les 298 producteurs des quatre bassins de production de l'île Maurice et leur cumul d'IFT (indice de fréquence de traitement).

Table 5. Crop rotation over a year observed among the 298 producers of the four production basins of Mauritius and their accumulation of FTI (Frequency of Treatment Index).

\begin{tabular}{|c|c|c|c|c|c|c|}
\hline $\begin{array}{l}\text { Bassins de production } \\
\text { Est }\end{array}$ & \multicolumn{5}{|c|}{ Successions culturales sur une année } & $\frac{\text { IFT }}{35}$ \\
\hline Est/Nord & Tomate & $\Rightarrow$ & Aubergine & & & 43 \\
\hline Est/Nord/Sud & Tomate & $\Rightarrow$ & Cucurbitacées & $\Rightarrow$ & Tomate & 53 \\
\hline Nord & Piment & & & $\Rightarrow$ & Tomate & 43 \\
\hline Nord & Lalo & & & & & 11 \\
\hline Nord & Cucurbitacées & $\Rightarrow$ & Tomate & $\Rightarrow$ & Haricot & 41 \\
\hline Centre-Ouest & Choux & $\Rightarrow$ & Choux & $\Rightarrow$ & Choux & 32 \\
\hline Centre-Ouest & Choux & $\Rightarrow$ & Légumes feuilles & $\Rightarrow$ & Haricot & 28 \\
\hline Centre-Ouest & Légumes feuilles & $\Rightarrow$ & Haricot & $\Rightarrow$ & Cucurbitacées & 29 \\
\hline Sud & Tomate & $\Rightarrow$ & Tomate & $\Rightarrow$ & Tomate & 48 \\
\hline
\end{tabular}


Enfin, concernant la gestion des fonds de cuve d'appareil après les traitements, près de $95 \%$ des producteurs déversent les eaux de rinçage sans aucune précaution et souvent près des sources d'approvisionnement en eau pour mieux rincer les appareils. Les substances actives des trois groupes présentent dans ces conditions des risques de transfert rapides de ces molécules vers les eaux souterraines.

\section{Discussion et conclusion}

Les producteurs de légumes mauriciens traitent de façon préventive les bioagresseurs de leurs cultures, avec pour conséquence des usages importants de pesticides. Ceux qui sont utilisés sont pour certains très dangereux pour l'environnement, notamment si de bonnes pratiques agricoles ne sont pas respectées. Selon la succession culturale, jusqu'à 53 traitements (insecticides/fongicides) peuvent être effectués annuellement. Ces pratiques répétées conduisent nécessairement à des impasses techniques de gestion de certains bioagresseurs, comme, par exemple, la mouche mineuse américaine (Liriomyza trifolii), qui occasionne des dégâts sur quasiment toutes les cultures légumières de l'île Maurice malgré l'usage quasi hebdomadaire d'insecticides. Ce bioagresseur, d'importance mondiale, est d'ailleurs connu pour sa résistance aux principaux insecticides (Hernandez et al., 2010). Des solutions autres que chimiques doivent être recherchées, d'autant que des bioagresseurs autrement plus impactant que cette mouche mineuse sont aux portes de l'île Maurice (ou ont déjà été détectés), comme la mouche orientale des fruits (Bactrocera dorsalis) ou encore une autre mineuse (Tuta absoluta), deux véritables fléaux à l'échelle internationale. Face à ces enjeux, seul le développement de systèmes de culture plus résilients peut être opposé. Pour y parvenir, deux principales actions doivent être menées à l'île Maurice. La première doit viser à mieux encadrer les usages des pesticides et la seconde, concomitante, à rechercher des solutions alternatives à ces usages pour développer des systèmes de culture durables.

\subsection{Mieux encadrer les usages des pesticides}

À l'île Maurice, seules les importations des pesticides sont réglementées, les usages restant à la discrétion des différentes parties prenantes qui doivent se conformer aux usages prévus par le fabriquant du pesticide. Dans la pratique, comme les producteurs et les conseillers souhaitent sécuriser les productions, les vendeurs de pesticides leur offrent un grand choix de molécules sans que les usages en soient réellement limités. Des détournements d'usage (application d'un pesticide sur une culture sur laquelle il n'est pas homologué) et les risques que cela comporte ont été observés, comme par exemple pour l'hexaconazole, fongicide autrefois utilisé pour lutter contre les maladies du blé et de l'orge (Ineris, 2016) et utilisé par les producteurs de légumes mauriciens. Par ailleurs, et considérant la législation française sur ces usages, certaines substances actives utilisées à l'île Maurice par les producteurs ne sont pas utilisées (ou ne le sont plus) en France, comme le propinebe ou encore le chlorfenapyr. Ce dernier est notamment connu pour sa très grande toxicité sur la faune et sa très lente dégradation dans l'écosystème (Albers et al., 2006). D'autres substances ont également été répertoriées lors de nos enquêtes alors que leur importation n'est plus autorisée sur l'île, comme le fenthion ou encore le formetanate. Tous ces usages sont symptomatiques d'un manque de connaissance des utilisateurs de ces pesticides. Une information suivie d'une réglementation sur leurs usages semble donc indispensable pour limiter, en amont, tous ces risques. L'outil d'aide à la décision «Phyto'Aide» peut dès lors devenir un outil d'accompagnement pertinent à la formation des agriculteurs. En effet, basés sur l'indicateur I-Phy - par ailleurs éprouvé dans de nombreux cas d'étude (Reus et al., 2002) -, cet outil d'aide à la décision propose des scores variant en fonction du pesticide, du type de sol le recevant et des conditions d'application. Ces scores I-Phy, comme la plupart des indicateurs, ne sont pas à prendre comme des valeurs absolues (Voltz et al., 2005) mais permettent de comparer les pesticides entre eux et, dans le cas de «Phyto'Aide», d'identifier les différents leviers pour limiter les risques de transfert des pesticides vers l'environnement. Cet outil d'aide à la décision devient dès lors un média de discussion entre le conseiller et le producteur pour accompagner le changement de pratiques ou la substitution de pesticides (Le Bellec et al., 2015).

\subsection{Rechercher des solutions alternatives à l'utilisation des pesticides}

De nouvelles stratégies de protection contre les bioagresseurs sont nécessaires d'une part pour limiter les pertes dues aux bioagresseurs et d'autre part pour réduire les investissements inutiles en intrants chimiques à forte empreinte environnementale (Carlton et al., 2012). La protection agroécologique, en favorisant le service écosystémique de régulation naturelle des bioagresseurs, peut permettre de diminuer durablement ces risques phytosanitaires. Face à ces enjeux, la re-conception des systèmes de production maraîchers mauriciens est interrogée, mais comment l'accompagner? Les deux premières étapes du cadre conceptuel de re-conception des systèmes - Efficiency, Substitution, Redesign (ESR)-, proposé par Hill et MacRae (1995), peuvent permettre de fixer des objectifs d'amélioration simples qui répondront rapidement aux contraintes mauriciennes. C'est la forme « faible» de modernisation écologique de l'agriculture proposée par Duru et al. (2014). Concrètement, dans notre cas d'étude, il s'agirait de favoriser des traitements curatifs plutôt que préventifs, en préférant notamment des pesticides présentant moins de risques pour l'environnement et la santé comme certains bio-pesticides, et d'accompagner les producteurs pour qu'ils respectent les bonnes pratiques agricoles comme le déclenchement des traitements basé sur des seuils de nuisibilité des bioagresseurs. Ces mesures faciles à mettre en œuvre permettraient d'atteindre le premier niveau du processus de re-conception du cadre ESR: l'efficience. Parallèlement, il s'agirait aussi d'utiliser des leviers techniques supplémentaires qui auraient des impacts rapides, pouvant ainsi et aussi répondre aux agriculteurs dont le foncier est incertain (étape de substitution du cadre ESR); comme, par exemple, utiliser des filets anti-insectes contre les mouches des cucurbitacées, utiliser des pièges à glue contre la mineuse des feuilles, des pièges à phéromones ou alimentaires pour lutter contre les mouches des légumes (Arida et al., 2013; Bruchon 
et al., 2015). Ces deux premières étapes contribueront à améliorer les systèmes de culture pas à pas, mais durablement. Le «Redesign», ultime étape du processus de Hill et MacRae (1995), induit nécessairement un changement de paradigme et viserait le développement de systèmes de culture agroécologiques dans lesquels les services écosystémiques remplaceraient totalement les intrants chimiques. Ces systèmes de culture mettraient au centre la restauration de la fertilité des sols, la lutte biologique par conservation des habitats, l'association avec l'élevage... (Bruchon et al., 2015). C'est la forme «profonde» d'une véritable transition agroécologique, pour laquelle Duru et al. (2014) proposent un cadre conceptuel d'accompagnement. Les acteurs sont au centre de leur démarche, ils participent à la construction du système agroécologique, mais aussi à la construction de systèmes résilients face à des contraintes climatiques de plus en plus prégnantes (Willaume et al., 2014). La territorialisation de tels systèmes agroécologiques doit donc être participative, holistique et transdisciplinaire, seule garantie de cette résilience. C'est à ce titre et au prix de tous ces efforts que la société mauricienne fera à nouveau confiance à la production fournie par ses producteurs de légumes.

Remerciements. Nous tenons à exprimer nos plus vifs remerciements aux producteurs interrogés qui ont permis ce travail, mais aussi aux conseillers du FAREI qui nous ont accompagnés sur le terrain. Ce projet a été cofinancé par l'Agence française de développement (AFD).

\section{Références}

Albers PH, Klein PN, Green DE, Melancon MJ, Bradley BP, Noguchi G. 2006. Chlorfenapyr and mallard ducks: overview, study design, macroscopic effects, and analytical chemistry. Environmental Toxicology and Chemistry 25: 438-445.

Arida GS, Punzal BS, Shepard BM, Rajotte EG. 2013. Sticky board traps for managing leafminer, Liriomyza trifolii (Burgess) (Diptera: Agromyzidae), infestation in onion (Allium cepa Linn.). Philippine Entomologist 27(2): 109-119.

Boodhoo I. 2014. National chemicals profile of the Republic of Mauritius. Port-Louis, (Mauritius): Ministry of Health and Quality of Life (MoHQL), 179 p.

Bockstaller C, Wohlfahrt J, Hubert A, Hennebert P, Zahm F, Vernier F, et al. 2008. Les indicateurs de risque de transfert de produits phytosanitaires et leur validation: exemple de l'indicateur I-Phy. Ingénieries 86: 103-114.

Bruchon L, Le Bellec F, Vannière H, Ehret P, Vincenot D, De Bon H, et al. 2015. Guide tropical-Guide pratique de conception de systèmes de culture tropicaux économes en produits phytosanitaires, Le Bellec F. éd. Cirad, 210 p.

Butler-Ellis MC, Swan T, Miller PCH, Waddelow S, Bradley A, Tuck CR. 2002. Design factors affecting spray characteristics and drift performance of air induction nozzles. Biosystems Engineering 82: 289-296.

Carlton RR, West JS, Smith P, Fitt BDL. 2012. A comparison of GHG emissions from UK field crop production under selected arable systems with reference to disease control. European Journal of Plant Pathology 133: 333-351.

Cilas C, Goebel FR, Babin R, Avelino J. 2015. Bioagresseurs des cultures tropicales face au changement climatique: quelques exemples. In: Torquebiau Emmanuel, ed. Changement climatique et agricultures $d u$ monde. Versailles (France): Éd. Quae, pp. $75-83$.

Davis AM, Thorburn PJ, Lewis SE, Bainbridge ZT, Attard SJ, Milla R, et al. 2011. Environmental impacts of irrigated sugarcane production: herbicide run-off dynamics from farms and associated drainage systems. Agriculture Ecosystems \& Environment 180: 123-135.

Duru M, Fares M, Therond O. 2014. Un cadre conceptuel pour penser maintenant (et organiser demain) la transition agroécologique de l'agriculture dans les territoires. Cahiers Agricultures 23: 84-95.

Forget PA, Prosper L, Savripène MA. 2016. [2016/09/13]. http:// www.lexpress.mu/article/274069/enquete-pesticides-ca-nousempoisonne.

Hernandez R, Harris M, Crosby K, Liu TX. 2010. Liriomyza (Diptera: Agromizydae) and parasitoid especies on pepper in the Lower Rio Grande Valley of Texas. Southwestern Entomologist 35: 33-43.

Hill SB, MacRae R. 1995. Conceptual frameworks for the transition from conventional to sustainable agriculture. Journal of Sustainable Agriculture 7: 81-87.

Ineris. 2016. [2016/09/20]. http://www.ineris.fr/substances/fr/sub stance/getDocument/3074.

Le Bellec F, Vélu A, Fournier P, Le Squin S, Michels T, Tendero A, et al. 2015. Helping farmers to reduce herbicide environmental impacts. Ecological Indicators 54: 207-216.

Lutchmeenaraidoo V. 2016. [2016/09/13]. http://www.lemauricien. com/article/discours-integral-du-budget-2015-2016-du-ministredes-finances-vishnu-lutcmeenaraidoo.

Reus J, Leendertse P, Bockstaller C, Fomsgaard I, Gutsche V, Lewis K., et al. 2002. Comparison and evaluation of eight pesticide environmental risk indicators developed in Europe and recommendations for future use. Agriculture Ecosystems \& Environment 90: 177-187.

Van der Werf HMG, Zimmer C. 1998. An indicator of pesticide environmental impact based on a fuzzy expert system. Chemosphere 36: 2225-2249.

Voltz M, Alix A, Barriuso E, Bedos C, Bonicelli B, Caquet T, et al. 2005. Devenir et transfert des pesticides dans l'environnement et impacts biologiques. In : Aubertot JN. Barbier JM, Carpentier A, Gril JN, Guichard L, Lucas P, Savary S, Voltz M, eds. Pesticides, agriculture et environnement - Réduire l'utilisation des pesticides et en limiter les impacts environnementaux. Expertise scientifique collective INRA-Cemagref, chapitre 3, pp. 1-219.

Willaume M, Audrey Rollin A, Casagrande M. 2014. Farmers in southwestern France think that their arable cropping systems are already adapted to face climate change. Regional Environmental Change 14: 333-345.

Citation de l'article : Le Bellec F, Scorbiac M, Sauzier J. 2017. Les pratiques phytosanitaires des producteurs de légumes de l'île Maurice : impacts et perspectives de changement. Cah. Agric. 26: 55001. 